\title{
ЛАЗЕРНАЯ ФЛУОРЕСЦЕНТНАЯ СПЕКТРОСКОПИЯ ВЕРХНИХ ВОЗБУЖДЕННЫХ СОСТОЯНИИ ХЛОРОФИЛЛОПОДОБНЫХ МОЛЕКУЛ
}

Изучение флуоресценции из верхних возбужденных состояний дает непосредственную информацию о быстрых безызлучательных процессах в молекулах и, несомненно, представляет большой интерес для установления общих закономерностей преобразования энергии электронного возбуждения. Нами впервые для хлорофиллоподобных молекул реализовано несколько вариантов двухквантового возбуждения флуоресценции из верхних возбужденных электронных состояний.

1. 3 амедленная флуоресценция из $S_{2}$-состояния ме т а л ло по р фи рин ов. При возбуждении непрерывными лазерами $\left(\mathrm{Ar}^{+}\right.$и $\left.\mathrm{He}-\mathrm{Ne}\right)$ обескислороженных растворов комплексов тетрафенилпорфина (ТФП) с $\mathrm{Mg}, \mathrm{Zn}, \mathrm{Lu}$ и тетрабензопорфина (ТБП) с $\mathrm{Zn}$ и $\mathrm{Cd}$ была зарегистрирована антистоксовая люминесценция в синей области спектра [ $\left.{ }^{1}\right]$. Коротковолновая флуоресценция (КВФ) спектрально совпадает с $S_{2} \rightarrow S_{0}$-флуоресценцией металлопорфиринов при прямом $S_{0} \rightarrow S_{2}$-возбуждении, но в отличие от последней деполяризована и исчезает при пуске воздуха в кювету. Зависимость интенсивности КВФ

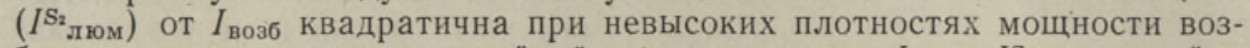
буждения и становится линейной при увеличении $I_{\text {возб} ; ~} I^{S_{1}}$ люм линейно

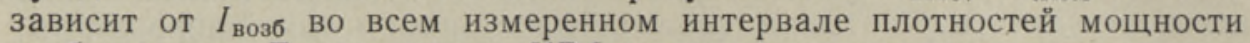
возбуждения. Длительность КВФ равна половине времени жизни $T_{1}$-состояния. Полученные данные показывают, что основным механизмом двухквантового возбуждения $S_{2}$-флуоресценции исследованных металлопорфиринов в обескислороженных растворах при плотностях мощности до $10^{20} \kappa в а н т /\left(c \mu^{2} \cdot c\right)$ является триплет-триплетная аннигиляция (ТТА).

Разработан метод определения вероятности образования синглетновозбужденной молекулы в результате аннигиляции двух триплетных молекул $\left(p_{a}\right)$, основанный на измерении отношения интенсивностей аннигиляционной замедленной флуоресценции (АЗФ) и быстрой флуоресценции (БФ) в условиях мощного импульсного возбуждения, когда процессы ТТА преобладают над процессами мономолекулярного распада триплетных состояний. Можно показать, что в этом случае выражение для $p_{a}$ имеет вид

$$
p_{a}=\frac{2 I_{\mathrm{A} 3 \Phi} / I_{\mathrm{Б} \Phi}}{\varphi_{T}\left[1+\left(I_{\mathrm{A} \Theta \Phi} / I_{\mathrm{Б} \Phi}\right)\right]} .
$$

Если в результате ТТА двух триплетных молекул может образоваться с вероятностью $p_{2}$ молекула в $S_{2}$-состоянии или с вероятностью $p_{1}$ молекула в $S_{1}$-состоянии, то

$$
\frac{p_{1}+p_{2}}{p_{2}}=\frac{p_{a}}{p_{2}}=\frac{I \stackrel{S_{1}}{S_{1}}}{I_{\mathrm{A} 3 \Phi}^{S_{2}}} \cdot \frac{I_{\mathrm{E} \Phi}^{S_{2}}}{I_{\mathrm{B} \Phi}^{S_{1}}} .
$$




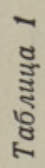

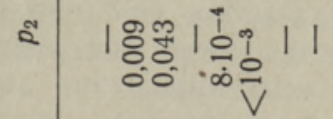

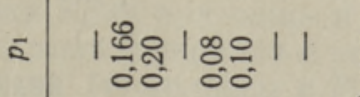

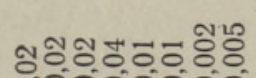

$\therefore \quad \mathrm{H} H \mathrm{H} H \mathrm{H}+\mathrm{H}+\mathrm{H}$

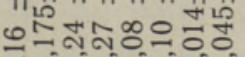
o.

* O.50.

- ज़

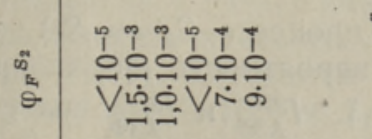

-

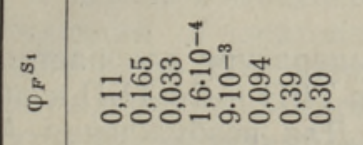

웅ㅇㅇㅇㅇㅁ웅요

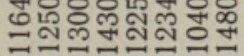

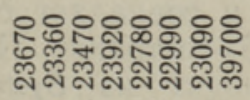

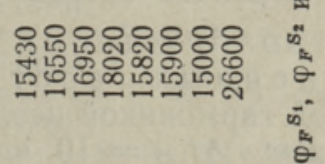

듭듭등등

列

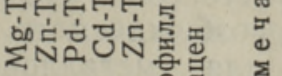

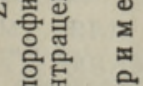

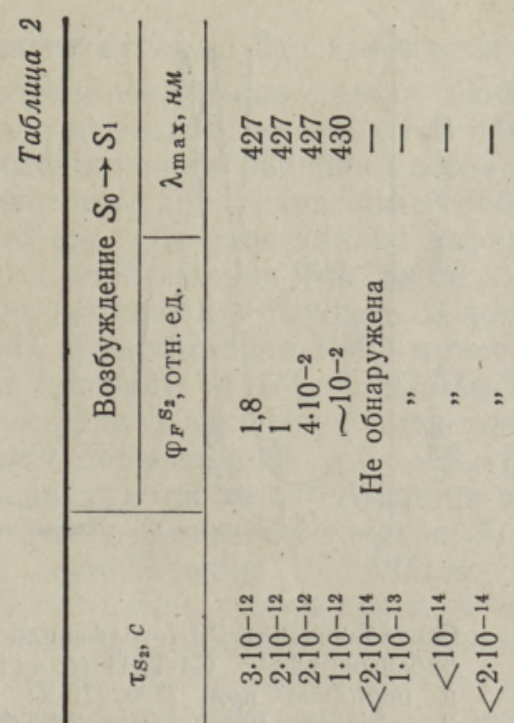

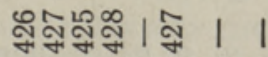

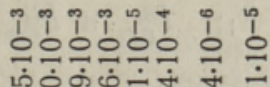

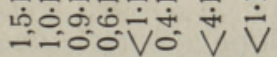

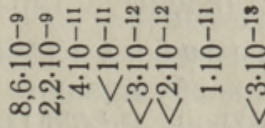

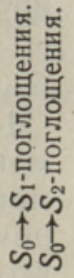

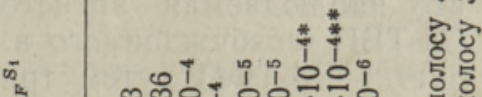

* 융의의의의

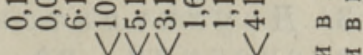

폴 落 瓷

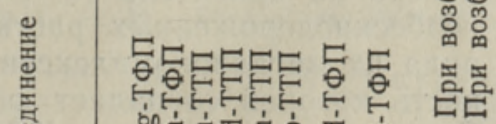

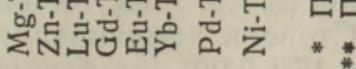




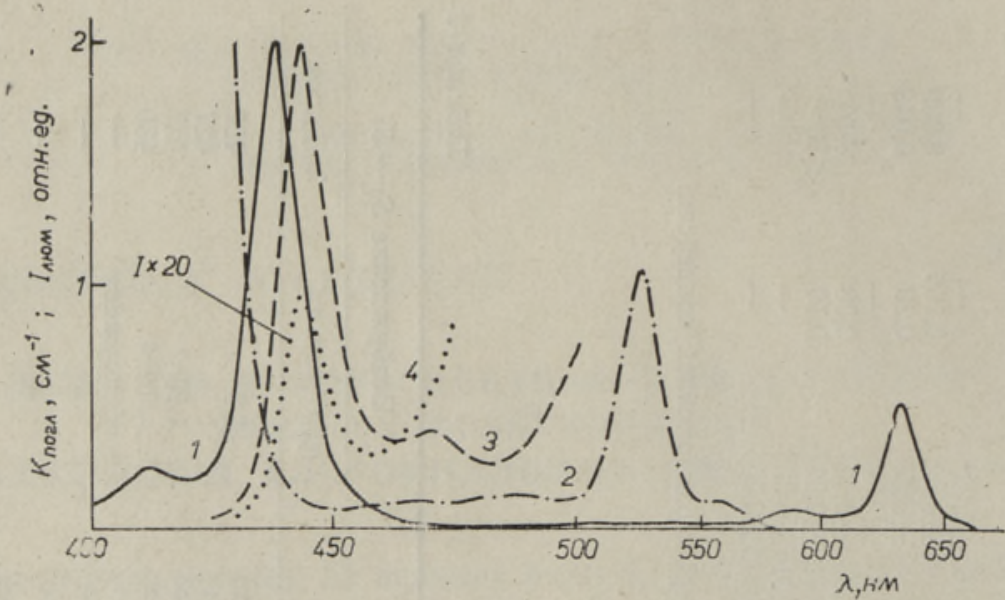

Сенсибилизация $\mathrm{Pd}$-тетрафенилпорфином $S_{2} \rightarrow S_{0}$-флуоресценции Cd-тетрабензопорфина. Сd-ТБП $\left(C=5 \cdot 10^{-6} M\right)$ и Рd-ТФП $\left(C=3,6 \cdot 10^{-5} M\right)$ в пиридине при $T=273 \mathrm{~K}: 1-$ спектр поглощения Сd-ТБП; 2 - спектр поглощения Рd-ТФП; 3 - спектр замедленной КВФ Cd-ТБП, сенсибилизованной $\mathrm{Pd}-\mathrm{TФП}\left(\lambda_{\text {возб }}=514,5\right.$ нм; $I_{\text {возб }}=$ $\left.=2,5 \cdot 10^{19} \kappa в а н т /\left(\mathrm{cm}^{2} \cdot c\right) ; \Delta \lambda_{\mathrm{per}}=0,2 \mu м\right) ; 4-$ КВФ в отсутствие $\mathrm{Pd}-\mathrm{T} П$ (при тех же $\left.\lambda_{\text {возб}}, I_{\text {возб и }} \Delta \lambda_{\text {per }}\right)$.

Здесь $p_{1}$ и $p_{2}$ - первичные вероятности (до процесса $S_{2} \rightsquigarrow S_{1}$ ), в то время как $p_{a}=p_{1}+p_{2}-$ окончательная вероятность образования $S_{1}$-состояния при ТТА (после процесса $S_{2} \rightsquigarrow S_{1}$ ). $I_{\text {АЗФ }}^{S_{1}}$ и $I_{\text {АЗФ }}^{S_{2}}-$ интегральные интенсивности АЗФ $S_{1} \rightarrow S_{0}$ и $S_{2} \rightarrow S_{0}$ при возбуждении $S_{0} \rightarrow S_{1} ; \quad I_{\text {БФ }}^{S_{1}}$ и $\underset{\text { БФ }}{S_{2}}-$ интенсивности БФ при прямом возбуждении $S_{0} \rightarrow S_{2}$.

Определены значения $p_{a}$ при комнатной температуре для растворов ТФП, Mg-ТФП, Zn-ТФП, Pd-ТФП в толуяле; Zn-ТБП и Cd-ТБП в пиридине; хлорофилла $a$ и антрацена в этаноле. Для возбуждения АЗФ применялись вторая $(\lambda=532$ нм) и третья $(\lambda=354$ нм) гармоники NdAИГ-лазера и лазер на красителе $(\lambda=575-595$ нм). Результаты представлены в табл. 1.

2. Сенсибилизация флуоресценции из $S_{2}-$ состояния. При возбуждении линией 514,5 нм аргонового лазера системы $\mathrm{Cd}-$ ТБП + Pd-TФП в пиридине (см. рисунок) регистрируется КВФ с максимумом при 443 нм. Спектр этой флуоресценции зеркально-симметричен спектру $S_{0} \rightarrow S_{2}$-поглощения Сd-ТБП и совпадает со спектром КВФ Сd-ТБП при прямом возбуждении в $S_{2}$-состояние. Показано, что наблюдаемая антистоксовая КВФ происходит из $S_{2}$-состояния $\mathrm{Cd}-\mathrm{TБ}$, возбуждаемого в результате гомогенной ТTA, и сенсибилизируется Рd-ТФП путем триплет-триплетного переноса энергии $\left[{ }^{2}\right]$.

3. Двухквантовое ступенчатое возбуждение флуоресценции из верхних возбужденных состояний. При мощном импульсном возбуждении второй гармоникой неодимового лазера ЛТИПЧ-8 $\left(\lambda=530 н м, E=2 м Д ж, \Delta t_{1 / 2}=10 н c\right)$, либо лазера на красителе $\left(\lambda=550-600\right.$ нм, $E=0,5$ мДж, $\left.\Delta t_{1 / 2}=10 \mu c\right)$ необескислороженных растворов ТФП, тетратолилпорфина (ТТП) и ряда их металлокомплексов обнаружена антистоксовая КВФ, длительность которой совпадает с длительностью возбуждаемого импульса. Степень поляризации КВФ составляет для металлопорфиринов $0,15 \pm 0,02$, что близко к теоретическому значению $1 / 7$ для этих соединений. Интенсивность антистоқсовой ҚВФ квадратично зависит от $I_{\text {возб }}$ 
в диапазоне, где $I_{\text {БФ }}^{S_{1}}$ линейно зависит от $I_{\text {возб. }}$ Все эти факты показывают, что наблюдаемая под действием импульсов лазера КВФ действительно принадлежит исследуемым металлопорфиринам и обусловлена двухквантовым ступенчатым возбуждением верхних состояний за время действия импульса лазера. Некоторые дополнительные соображения о механизме возбуждения можно сделать, сопоставляя характеристики КВФ при одно- и двухквантовом возбуждении (см. табл. 2). Для металлопорфиринов с приблизительно одинаковым выходом $S_{2}$-флуоресценции (комплексы с $\mathrm{Mg}, \mathrm{Zn}, \mathrm{Lu}, \mathrm{Cd}$ ) антистоксовая КВФ имеет боль-

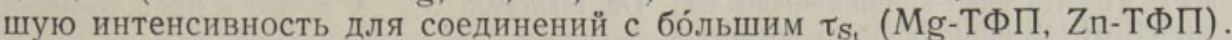
Это показывает, что под действием мощного излучения антистоксовая КВФ возбуждается в основном за счет переходов $S_{0} \rightarrow S_{1} \rightarrow S_{n}\left[{ }^{3}\right]$.

Для комплексов с $\mathrm{Pd}$ и $\mathrm{Ni} S_{2}$-флуоресценция не наблюдается ни при прямом, ни при ступенчатом возбуждении. Квантовый выход $S_{1}$-флуоресценции $\mathrm{Pd}-\mathrm{TФП} \mathrm{постоянен} \mathrm{при} \mathrm{возбуждении} \mathrm{в} \mathrm{области} \mathrm{полос}$ $S_{0} \rightarrow S_{1}$-поглощения и падает в 1.5 раза при возбуждении в полосу $S_{0} \rightarrow S_{2}$ как при $293 \mathrm{~K}$, так и при $77 \mathrm{~K}$. Квантовый выход фосфоресцен-

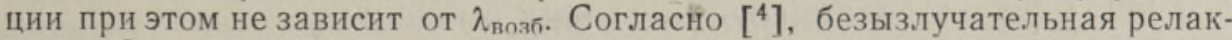
сация $S_{2}$-состояния металлопорфиринов, не имеющих ниже $S_{2}$-состояния уровней, связанных с возбуждением электронов иона металла, осуществляется путем внутренней конверсии $S_{2} \rightsquigarrow S_{1}$. Поэтому, учитывая данные пикосекундной спектроскопии $\left[{ }^{5,6}\right]$, можно предложить следующую схему релаксашии энергии в комплексах с $\mathrm{Ni}(\mathrm{II})$ и $\mathrm{Pd}(\mathrm{II})$. B случае комплексов с $\mathrm{Ni}(\mathrm{II})$ как из $S_{2-}$, так и из $S_{1}$-состояния происходит быстрый безызлучательный перенос энергии в синглетное ${ }^{1} B \cdot q$-состояние иона $\mathrm{Ni}(\mathrm{II})$. Константы скорости переноса энергии из $S_{9}$ - и $S_{1}$-состояний превышают $5 \cdot 10^{13}$ и $3 \cdot 10^{12} c^{-1}$ соответственно. Из ${ }^{1} B_{1 g}$-состояния $3 а$ $10^{-11} c$ реализуется переход в ${ }^{3} B_{1 g}$-состояние, время жизни последнего составляет 250 nc.

В случае комплексов с $\mathrm{Pd}(\mathrm{II})$ vровни ${ }^{1} B_{1 g}$ и ${ }^{3} B_{1,}$, связанные с возбуждением $d$-электронов иона $\mathrm{Pd}(\mathrm{II})$, по-видимому, расположены между $S_{2}$ и $S_{1} \pi$-электронными уровнями порфинного кольша. После возбуждения в $S_{2}$-состояние реализуется (с константой $>10^{14} c^{-1}$ ) внутримолекулярный пепенос энергии на ${ }^{1} B_{1 g}$-уровень, с которого затем происходит перенос на $S_{1}{ }^{\pi \pi}$-уровень. Олнако скорость интеркомбинационной конверсии ${ }^{1} B_{1,} \rightsquigarrow{ }^{3} B_{1 \text { r }}$ в случае $\mathrm{Pd}(\mathrm{II})$ несомненно больше, чем в случае $\mathrm{Ni}(\mathrm{II})$, и сопоставима со скоростью ${ }^{1} B_{1,} \rightarrow S_{1}{ }_{1} \pi$-переноса энергии. В результате часть молекул не попадает в $S_{1}{ }^{\pi \pi}$-состояние. а переходит в ${ }^{3} B_{1}$-состояние. откуда затем путем разрешенного по спину триплет-триплетного переноса энергии переходит в $T_{1}{ }_{1} \pi$-состояние.

Л.И Т Е Р А Т У Р А.

1. С тельм ах Г. Ф., Цв и рко М. П., Оптика и спектроскопия, 49, вып. $3,511-516$ (1980)

2. С тельм х Г. Ф., Цв и рко М. П., Оптика и спектроскопия, 50, вып. 5, 998-1001 (1981).

3. С тельм а Г. Ф., Цв и р ко М. П., Оптика и спектроскопия, 48 , вып. $1,185-188$ (1980).

4. Tsvirko. M. P., Ste $1 \mathrm{makh}$, G. F., Py a tosin, V. E. et al., Chem. Phys. Lett., 73, № $1,80-83$ (1980).

5. Kobayashi. T., Straub, K. D., Rentzepis, P. M., Photochem. Photobiol., 29, 925-931 (1979).

6. Chirvonyi, V. S., Dzhagarov, B. M., Timinskii, Yu. V., Gurinov i c h, G. P., Chem. Phys. Lett., 70, № 1, 79-83 (1980).

Научно-исследовательский институт прикладных физических проблем

при Белорусском государственном университете 


\section{KLOROFULLISARNASTE MOLEKULIDE OLEMISTE ERGASTUSOLEKUTE LASERFLUORESTSENTSSPEKTROSKOOPIA}

On näidatud, et pidevatoimeliste ja impulsslaseritega saab klorofüllisarnaseid molekule kahekvandilise järjestikuse ergastusega viia ülemistesse ergastusolekutesse kas astmelise ergastusega vaheolekust $S_{1}$ või triplett-triplett-annihilatsiooni teel. Metalloporfüriinide $S_{2}$-oleku fluorestsentsi on sensibiliseeritud triplett-triplett-energiaülekande ja järgneva triplett-triplett-annihilatsiooni teel. On määratud $S_{1^{-}}$ja $S_{2}$-oleku tekke tõenăosus triplett-triplett-annihilatsiooni korral.

M. P. TSVIRKO, G. F. STELMAKH

\section{LASER FLUORESCENCE SPECTROSCOPY OF UPPER EXCITED STATES OF CHLOROPHYLL-LIKE MOLECULES}

A consecutive two-photon excitation of upper excited singlet states of chlorophyll-like molecules, both by stepwise excitation through an intermediate $S_{1}$ level and by triplettriplet annihilation, has been produced by the continuous and pulsed laser excitation in $S_{0} \rightarrow S_{1}$ absorption band. The sensitization of the $S_{2} \rightarrow S_{0}$ fluorescence of metalloporphyrins by means of triplet-triplet energy transfer and a subsequent triplet-triplet annihilation has been realized. The primary population probabilities of $S_{1}$ and $S_{2}$ states after the triplet-triplet annihilation have been measured. The pathways and mechanisms of the excited state relaxation in $\mathrm{Ni}(\mathrm{II})$ and $\mathrm{Pd}(\mathrm{II})$ porphyrins have been discussed. 